\title{
Impact of Comprehensive Medication Management on Hospital Readmission Rates
}

\author{
Holly Budlong, PharmD, PhD, ${ }^{1}$ Amanda Brummel, PharmD, BCACP,, \\ Adam Rhodes, $\mathrm{MS}^{3}$, and Hannah Nici, PharmD ${ }^{2}$
}

\begin{abstract}
In 2012, the Fairview Health System implemented a formal care transitions process that included referrals to outpatient services provided by medication therapy management (MTM) pharmacists, among other clinical services. This analysis evaluates the impact of the MTM-provided comprehensive medication management (CMM) service on readmission rates. Retrospective electronic medical record (EMR) data were used to identify hospital admissions between December 1, 2012, and July 31, 2015. Thirty- and 60-day readmission rates were calculated in both a CMM and comparator cohort. Readmission rates also were stratified by readmission risk category. A total of 43,711 patients, contributing 57,673 hospitalizations, were included in the analysis. Of those, 1291 hospitalizations had a CMM visit within 30 days of discharge (median 6 days) and were considered the CMM cohort. Patients who received a CMM visit had a significantly lower rate of 30-day readmissions (8.6\% vs. $12.8 \%, P<0.001$ ). The 60 -day readmission rate remained lower among CMM patients but did not reach statistical significance $(15.6 \%$ vs. $17.6 \% ; P=0.0528)$. When patients in each cohort were stratified by readmission risk category, the CMM cohort had a statistically significant lower rate of 30-day readmission in the highest risk groups (Average: $7.1 \%$ vs. $9.5 \%, P=0.025$; Elevated: $9.9 \%$ vs. $21.4 \%, P<0.001$; High: $18.3 \%$ vs. $35.9 \%, P<0.001$; Extreme: $36.4 \%$ vs. $77.7 \%, P=0.006$ ). CMM performed by an MTM pharmacist reduces the rate of readmission at 30 days post discharge and may have the largest impact among patients at highest risk of readmission.
\end{abstract}

Keywords: drug use, population health, risk stratification, quality measures, evidence-based care

\section{Introduction}

I MPROVING CARE TRANSITIONS in order to reduce hospital readmissions has become a focus of most health systems in recent years. A transition of care is "any instance in which a patient moves from one health care setting to another"; however, the transition from the hospital is most commonly studied. ${ }^{1}$ This is in large part because of the recent changes in the Centers for Medicaid \& Medicare Services (CMS) reimbursement for hospital readmissions. ${ }^{2}$ The Hospital Readmissions Reduction Program reduces payments for all Medicare patients to hospitals that have above-average 30day readmission rates for certain disease states. The national 30-day readmission rate for Medicare-reimbursed hospitals for the measurement period ending June 30, 2016 was $15.3 \% .^{3}$ In 2015, a total of 2597 hospitals were fined for readmission rates that were above the threshold. ${ }^{4}$ In 2017 , $79 \%$ of hospitals will be penalized with penalties expected to total \$528 million. ${ }^{5}$
The financial pressure from CMS has resulted in many different programs designed to reduce readmissions. ${ }^{6-8} \mathrm{~A}$ Minnesota program, Reducing Avoidable Readmissions Effectively (RARE) campaign, sought to decrease avoidable readmissions by 4000 over the course of 3 years. ${ }^{6}$ The RARE campaign focused on 5 areas: comprehensive discharge planning, medication management, patient and family engagement, transition care support, and improvement in transition communications. During the trial period, 7975 readmissions were prevented, exceeding the program's goal.

Boston University Medical Center developed Project Re-Engineered Hospital Discharge (Project RED). ${ }^{7}$ This focused on different "Components of Re-Engineered Discharge" to facilitate an orderly discharge. Many different techniques were utilized, including discharge planning, scheduling follow-up appointments and tests, specific focus on medications and medication education, medication reconciliation, a contingency plan once a patient arrived home, and a phone call within 3 days of discharge. This team-

\footnotetext{
${ }^{1}$ Health Outcomes, Fairview Pharmacy Services, Minneapolis, Minnesota.

${ }^{2}$ Medication Therapy Management, Fairview Pharmacy Services, Minneapolis, Minnesota.

${ }^{3}$ Population Health Analytics, Fairview Health Services, St. Paul, Minnesota.
} 
based method decreased readmissions by $30 \%$ when compared to the baseline readmission rate.

Although the health system programs mentioned previously all included a medication management component, the overall benefit of pharmacist interventions has been inconsistent resulting in inconclusive consensus regarding the usefulness of services offered by clinical pharmacists. ${ }^{9,10}$ Although readmission reduction programs often include clinical pharmacists, the services offered vary widely and may occur in combination with other interventions.

Prior evaluations of the impact of pharmacist-provided medication therapy management (MTM) services within the Fairview system have consistently demonstrated a significant impact on patient outcomes ${ }^{11-13}$; however, the impact of these services on hospital readmissions has not been evaluated.

This study examines the impact of comprehensive medication management (CMM) on readmission rates within a health system that utilizes MTM pharmacists who follow a consistent and well-defined patient care process.

\section{Methods}

\section{Setting}

Fairview Health Services, in partnership with the University of Minnesota, is a network of 7 hospitals, 45 primary care clinics, 55+ specialty clinics, and 30+ retail pharmacies that serves the greater Minneapolis-St. Paul area as well as communities throughout Minnesota and the upper Midwest. The not-for-profit corporation is one of the largest health care providers in Minnesota. Fairview's MTM program, developed in partnership with the University of Minnesota, College of Pharmacy, has been providing CMM for almost 20 years. Currently, the program includes 25 MTM pharmacists (approximately 17.5 full-time equivalents) working in 33 locations, including both primary care/internal medicine and specialty clinics.

In 2012, Fairview implemented a formal transitions of care process. Patients discharged from Fairview hospitals are referred to outpatient services provided by MTM pharmacists, behavioral health specialists, and care coordinators. Specific services are selected based on various patient clinical characteristics. The program was initially implemented in 2 hospitals, was expanded to other facilities throughout 2012 and 2013, and is currently available in all but 1 Fairview hospital.

Transitions of care visits are considered a health system benefit, so all patients who are discharged from one of Fairview's hospitals are eligible for 2 visits by an MTM pharmacist at no charge, regardless of insurance coverage. The visits must be within 30 days of hospital discharge.

During the time period of this evaluation, referrals specifically to an MTM pharmacist were based on specific chronic disease state diagnoses, number of chronic medications, utilization data, and documented medication nonadherence (Table 1). For patients who meet the referral criteria, an alert is triggered in their electronic medical record (EMR) recommending a care coordinator and/or inpatient pharmacist place a referral for a CMM visit. The MTM coordinators receive the referral and schedule a CMM visit as soon as possible post discharge. Additionally, a care coordinator from the patient's primary care clinic calls the patient within 48 hours of discharge and can place a CMM referral if necessary based on their assessment during the call.

At Fairview, MTM pharmacists utilize the principles of CMM described by Cipolle et al, whereby each of the patient's medications is evaluated for indication, efficacy, safety, and convenience. ${ }^{14,15}$ Each CMM encounter follows a systematic process designed to identify and resolve drug therapy problems and promote optimal patient outcomes. The pharmacist focuses on the patient in a comprehensive manner and assesses all of the patient's diseases and corresponding medications. Medication reconciliation also is performed at each visit and discrepancies are brought to the attention of the primary care provider.

For patients with a primary care provider within the Fairview health system, MTM pharmacists also have the ability to initiate, discontinue, or adjust certain medications included in their collaborative practice agreement (CPA). The CPA covers more than 15 disease states including heart failure, chronic obstructive pulmonary disease, diabetes, hyperlipidemia, hypertension, urinary incontinence and osteoporosis. During each CMM visit, pertinent medical information is collected, organized, and documented; medications are assessed; and a care plan is developed with the patient. All information related to the visit is documented in the EMR.

Typically, CMM visits are provided by the MTM pharmacist located in the same clinic as the patient's primary care provider. This allows the pharmacist and the patient to interact face-to-face. Patients are encouraged to have the visits in the clinic; however, phone or virtual visits are offered for patients who are unwilling or unable to travel to the clinic. Pharmacists have a dedicated schedule of 30- to 60-minute appointments allocated for each patient. Hourlong appointments usually are reserved for initial visits; however, follow-up visits may be up to an hour long at the provider's discretion.

\section{Study design and outcomes}

The objective of this evaluation was to determine whether or not a CMM visit with an MTM pharmacist within 30 days of hospital discharge decreased readmissions at 30 days post discharge when compared to a complexity-matched group of patients who did not receive a CMM visit. Secondary analyses were conducted to evaluate the 60-day readmission rates and readmission rates among different readmission risk categories.

This retrospective analysis used EMR data to identify patients with at least 1 Fairview hospital admission between December 1, 2012, and July 31, 2015. Patients were required to be 18 years of age or older and were excluded if the primary discharge diagnosis was pregnancy or issues relating to pregnancy, they died during the hospital stay, were discharged to any facility other than home (eg, rehabilitation facility, long-term care facility, prison), or discharged with a planned readmission to any health care facility.

Readmission rates were calculated in both the CMM and comparator cohort using proportions of patients readmitted to the hospital within 30 or 60 days post discharge. Readmission rates also were calculated for each risk category. Readmission risk categories include Low, Average, Elevated, High, Very 
Table 1. Comprehensive Medication Management Referral Criteria for Transitions of Care Program

\begin{tabular}{|c|c|c|c|c|}
\hline \multicolumn{2}{|c|}{ Chronic disease state \& number of chronic medications } & \multicolumn{3}{|c|}{ Medication utilization } \\
\hline $\begin{array}{l}\text { Newly diagnosed: } \\
\text { Diabetes mellitus } \\
\text { Heart failure } \\
\text { Chronic obstructive } \\
\text { pulmonary disease } \\
\text { Acute myocardial } \\
\text { infarction } \\
\text { AND } \\
5+\text { chronic medications }\end{array}$ & $\begin{array}{l}\text { Hospital admission or emergency } \\
\text { department visit within } \\
\text { prior } 6 \text { months } \\
A N D \\
10+\text { chronic medications }\end{array}$ & $\begin{array}{l}\text { Use of outpatient } \\
\text { rifaximin } \\
\text { or lactulose }\end{array}$ & $\begin{array}{l}\text { Use of outpatient } \\
\text { oral chemotherapy }\end{array}$ & $\begin{array}{l}\text { Suspected or } \\
\text { documented } \\
\text { medication } \\
\text { nonadherence }\end{array}$ \\
\hline
\end{tabular}

An electronic medical record alert recommending a medication therapy management referral was triggered when a patient met one of the criteria.

High, and Extreme. Inclusion in each category was determined by an internal algorithm that utilizes 8 key predictors of hospital readmission (age, length of stay, location, acuity, admitting diagnosis, cancer treatment, birth, and 6-month hospital history). The algorithm has been validated using more than 60,000 hospital discharges and is currently being implemented as an assessment tool for patient discharge planning within Fairview.

The intervention (CMM) cohort included any patient with a documented CMM visit from a Fairview MTM pharmacist within 30 days post hospital discharge. All CMM visit types were included: face-to-face, office, telephonic, and virtual. The comparator cohort included patients discharged with a Diagnosis-Related Group included in the CMM cohort but who had no evidence of a CMM visit at any time in the evaluation period. Because patients could receive their CMM visit up to 30 days post discharge, a sensitivity analysis also was conducted limiting the CMM cohort to patients who received a CMM visit within 7 days of discharge. This sensitivity analysis was intended to ensure that results were consistent when patients had a longer duration of time to readmit after their CMM visit.

\section{Statistical analysis}

Thirty- and 60-day readmission rates were calculated for each cohort. The denominator was comprised of discharges that met all inclusion criteria, and the numerator was comprised of the number of discharges for which a readmission occurred within the respective time frame. Readmission rates also were compared within each readmission risk category stratum. All rates were compared using a proportion $Z$ test with $\alpha$ set at 0.05 . Statistical analyses were conducting using MiniTab 17, version 17.3.1 (MiniTab Inc., State College, PA). Institutional Review Board approval was requested from the University of Minnesota and expedited approval was granted prior to the data request.

\section{Results}

In total, 43,711 patients, contributing 57,673 hospitalizations, were included in the analysis. Of those, 1291 hospitalizations had a CMM visit within 30 days of discharge. Patient characteristics of the 2 groups were similar (Table 2), with the exception of age. On average, patients who received a CMM visit were older than patients in the comparator cohort (63.1 vs. 55.6 years, respectively). The majority of patients in both cohorts were categorized with a risk of readmission as Average. In the CMM cohort, the median time from discharge to the first CMM visit was 6 days (interquartile range $3-12$ ).

\section{Readmission rates}

At 30 days post discharge, patients who received a CMM visit had a significantly lower rate of readmissions compared to the comparator cohort (4.2\% lower, $P<0.001$; Fig. 1$)$. The 60-day readmission rate remained lower among CMM patients compared to the comparator cohort but did not reach statistical significance ( $2 \%$ lower; $P=0.0528)$.

When patients in each cohort were stratified by readmission risk, the CMM cohort had a lower rate of 30-day readmission across all categories and reached statistical significance in the Average, Elevated, High, and Extreme groups (Fig. 2). The CMM cohort still had significantly lower rates of 60-day readmission across all groups, except Low, and reached statistical significance for Elevated, High, Very High, and Extreme readmission risk groups (Fig. 3). In addition, as the risk of readmission increased, the difference between the CMM cohort and comparator cohort readmission rates generally increased.

In the CMM cohort, $60 \%$ of patients received their CMM visit within 7 days of hospital discharge. To ensure the impact of CMM visits on 30-day readmissions among patients with

TAble 2. Cohort Demographic Information

\begin{tabular}{lcc}
\hline & $\begin{array}{c}\text { CMM } \\
\text { cohort } \\
\text { Patients (N) }\end{array}$ & $\begin{array}{c}\text { Comparator } \\
\text { cohort } \\
42,523\end{array}$ \\
\hline Hospitalizations, N & 1188 & 56,382 \\
Age, Average (SD) & $63.1(14.0)$ & $55.6(17.3)$ \\
Female, & $50.2 \%$ & $51.6 \%$ \\
Readmission Score, & $2.5(1.5)$ & $2.3(1.5)$ \\
$\quad$ Average (SD) & & \\
Readmission Risk & & \\
$\quad$ Category, N (\%) & $233(18.0 \%)$ & $18,147(32.2 \%)$ \\
Low & $675(52.3 \%)$ & $24,094(42.7 \%)$ \\
Average & $273(21.1 \%)$ & $9771(17.3 \%)$ \\
Elevated & $60(4.6 \%)$ & $2526(4.5 \%)$ \\
High & $39(3.0 \%)$ & $1153(2.0 \%)$ \\
$\quad$ Very High & $11(0.9 \%)$ & $691(1.2 \%)$ \\
$\quad$ Extreme &
\end{tabular}

CMM, comprehensive medication management; SD, standard deviation. 


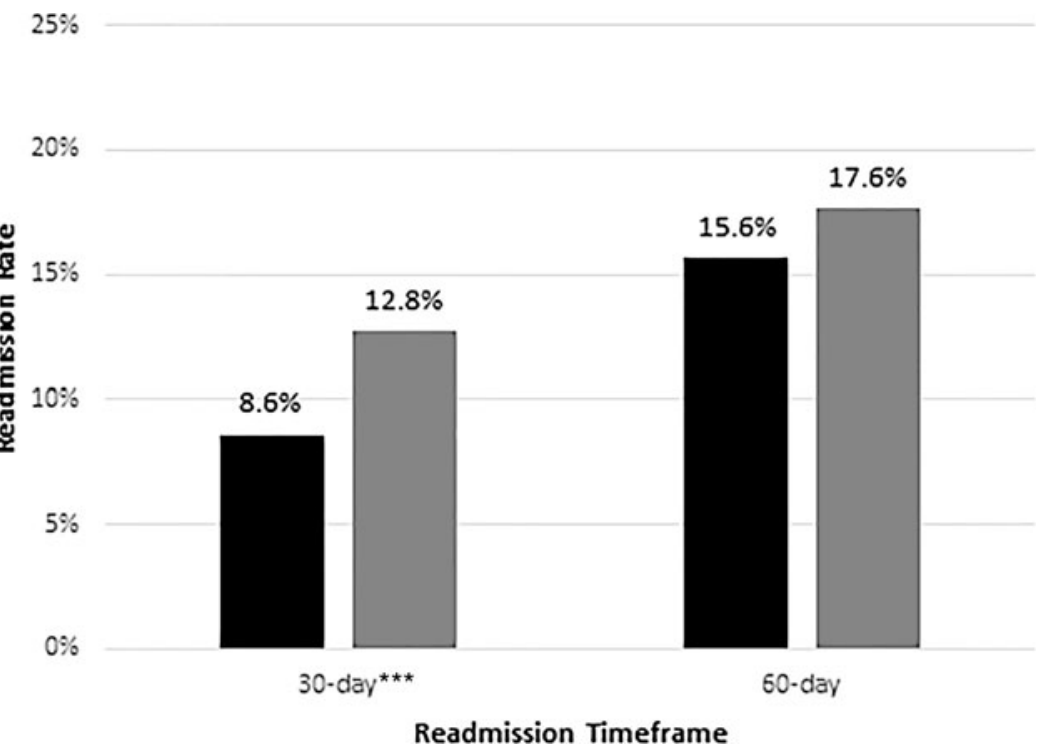

FIG. 1. Thirty- and 60-day readmission rates for comprehensive medication management and comparator cohorts. CMM cohort. Comparator cohort $* * * P$-value $<0.001$. CMM, comprehensive medication management.

longer follow-up time was consistent with the overall findings, a sensitivity analysis was conducted limiting the CMM group to those who had a visit within 7 days of discharge. The results of this analysis were consistent with the primary objective and the CMM cohort had a significantly lower rate of 30 -day readmissions $(10.2 \%$ vs. $12.8 \%, P=0.020)$.

\section{Discussion}

This study adds to the body of literature demonstrating that pharmacists are an integral member of the care team and can contribute to reductions in hospital readmissions by providing CMM. In addition, the data for 60-day readmissions suggests that the impact of MTM pharmacistprovided CMM persists beyond an immediate effect.

The mixed results regarding the impact of MTM services on readmission rates is likely related to the variability in the way MTM services are delivered across the health care industry. The results identified in this study are likely attrib- uted to the comprehensive patient care process that is consistently implemented across all Fairview MTM practitioners and the collaboration and coordination of the MTM practitioners with the health care team. Therefore, the significant decrease in readmissions identified here is difficult to compare to other studies that have evaluated the effectiveness of pharmacist interventions because of the variability in the definition of CMM and the services provided.

The results of this study have prompted enhancements and modifications across the Fairview transitions of care program. First, the findings suggest that a larger population of patients may benefit from MTM services. During the study period, only 1291 discharge episodes out of 57,673 had a completed CMM visit by an MTM pharmacist after discharge. The study team is evaluating gaps in the current referral process and making recommendations to streamline referral placement once appropriate patients are identified. One finding thus far suggests that the referral alert does not

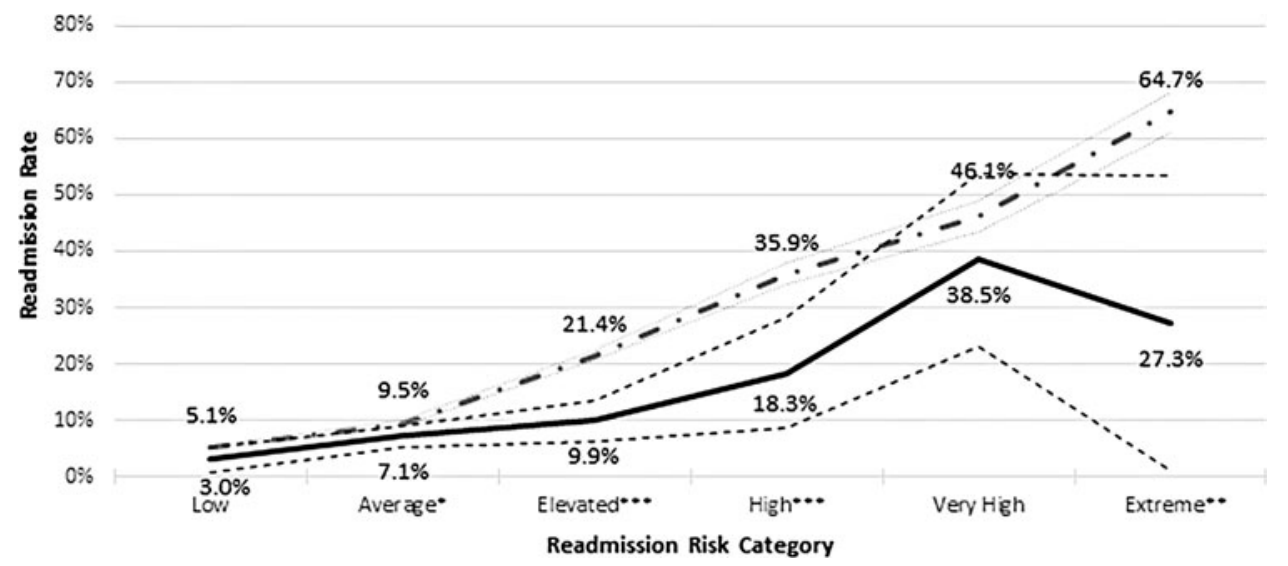

FIG. 2. Thirty-day readmission rates stratified by readmission risk category.

CMM cohort. - ..... . CMM cohort CI. - - Comparator cohort. Comparator cohort CI. $* * * P$ value $<0.001$; $* * P$ value $<0.01$; $* P$ value $<0.05$. CI, confidence interval; CMM, comprehensive medication management. 


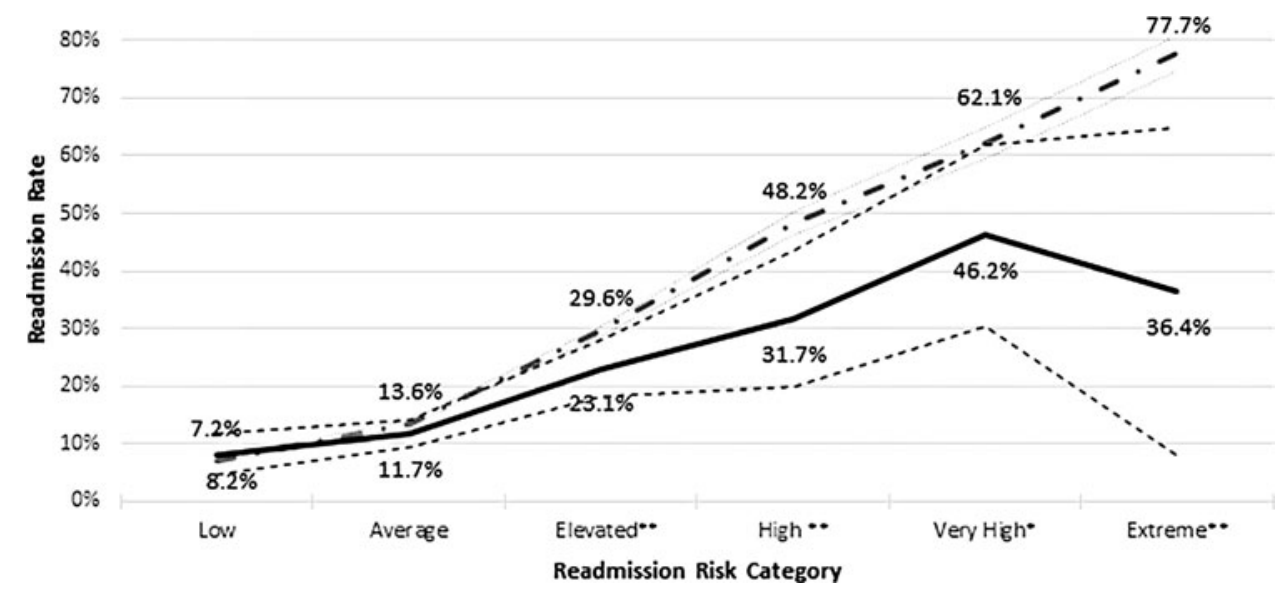

FIG. 3. Sixty-day readmission rates stratified by readmission risk category. cohort CI. - - Comparator cohort. ……........ Comparator cohort CI. $* * P$ value $<0.01$; $* P$ value $<0.05$. CI, confidence interval; CMM, comprehensive medication management.

fit into the care coordinator workflow well. The team is currently investigating timing of alert notifications to the care coordinators and determining better ways to incorporate the referral alert to subsequently increase CMM referral placements. Currently only a few inpatient pharmacy departments have the referral alert incorporated into their workflow. The study team also plans to expand alert notifications to additional inpatient pharmacy units.

Given the volume increase that improving the CMM referral placement would create, the referral criteria will be refined to include not only medication and disease state elements but also readmission risk based on the calculated readmission risk score. This will allow more efficient identification of patients who could benefit most from CMM. A dashboard also will be created to more easily identify the number of referral alerts being generated and those for which a referral is actually being placed. In addition, increased awareness of MTM services for both patients and care team colleagues is necessary to ensure adherence to referral recommendations.

Overall, as other health systems engage pharmacists in transitions of care programs, it is important to provide comprehensive services. In addition, to ensure programs are comparable across the health care industry, a consistent definition and delivery of CMM is needed.

\section{Limitations}

One limitation of this study is the varied time frame with which patients received their CMM visit. The goal of the program was for patients with a referral to be seen within 7 days of discharge. Although the majority of patients included in the CMM group were able to complete their visit within 6 days of being discharged, there were patients included in the cohort who received their CMM visit as late as 30 days. The sensitivity analysis, which included only patients who received a CMM visit within 7 days of discharge, produced similar results to the primary analysis, with patients who received CMM having a significantly lower rate of 30-day readmissions.
Another limitation is the inconsistency of the MTM referral process. Referrals are not placed consistently on discharge, and patients who may benefit from MTM services may not have had a referral placed. Although an alert was triggered for all patients meeting the MTM referral criteria, practitioners can bypass the alert without a referral being placed. In addition, a referral can be placed without the presence of an alert. Though a nurse could place a referral during the 48-hour postdischarge call, this is a delay of MTM services that may be detrimental to the patient, especially those at highest risk for readmission. Although inconsistencies are a limitation of the program overall, in regard to this analysis, MTM-eligible patients who never received a referral contributed to the control group, allowing for a more similar patient distribution between the 2 groups. Both patient groups also were eligible to receive other outpatient health care services, though it is likely patients in the 2 groups had similar interactions with other health care professionals.

The data for this research includes episodes of care exclusively delivered by Fairview and recorded in the EMR. Patients could have been hospitalized at other health systems; however, the impact of this is likely to be nondifferential.

\section{Conclusion}

CMM performed by an MTM pharmacist reduces rates of readmission at 30 and 60 days post discharge when compared to a complexity-matched control group. In addition, CMM may have the largest impact among patients at highest risk of readmission. Further studies should focus on the transitions of care team as a whole and the differences between CMM visits conducted in the office, over the phone, and virtually.

\section{Acknowledgments}

The authors would like to acknowledge Wendy St. Peter, Brittany Hogan, and Sirikan Rojanasarot for their assistance with this project including data gathering and the early analysis of the program results. 


\section{Author Disclosure Statement}

The authors declare that there are no conflicts of interest. The authors received no financial support for the research, authorship, and/or publication of this article.

\section{References}

1. Shekelle PG, Wachter RM, Pronovost PJ, et al. Making health care safer II: an updated critical analysis of the Evidence for patient safety practices. Evidence Report/ Technology Assessment No. 211. Rockville, MD: Agency for Healthcare Research and Quality, 2013.

2. Centers for Medicare \& Medicaid Services. Readmissions Reduction Program (HRRP). Last modified November 30, 2017. www.cms.gov/medicare/medicare-fee-for-service-payment/ acuteinpatientpps/readmissions-reduction-program.html Accessed December 29, 2017.

3. Data.Medicare.gov. Unplanned Hospital Visits-National. Updated December 21, 2017. https://data.medicare.gov/ Hospital-Compare/Hospital-Returns-National/cves-xecj Accessed December 29, 2017.

4. Kaiser Health News. Medicare readmission penalties hit new high. 2016. http://khn.org/news/more-than-half-of-hospitals-tobe-penalized-for-excess-readmissions/ Accessed March 1, 2017.

5. Boccuti C, Casillas G. Aiming for Fewer Hospital U-Turns: The Medicare Hospital Readmission Reduction Program. 2017. www.kff.org/report-section/aiming-for-fewer-hospital-u-turnsthe-medicare-hospital-readmission-reduction-program-issuebrief/\#endnote_link_211172-6 Accessed September 14, 2017.

6. Institute for Clinical Systems Improvement; Minnesota Hospital Association; Stratis Health. Reducing Avoidable Readmissions Effectively. www.rarereadmissions.org/index. html Accessed February 6, 2015.

7. Jack BW, Chetty VK, Anthony D, et al. A reengineered hospital discharge program to decrease rehospitalization: a randomized trial. Ann Intern Med 2009;150:178-187.
8. Trang J, Martinez A, Aslam S, Duong MT. Pharmacist advancement of transitions of care to home (PATCH) service. Hosp Pharm 2015;50:994-1002.

9. Viswanathan M, Kahwati LC, Golin CE, et al. Medication therapy management interventions in outpatient settings: a systematic review and meta-analysis. JAMA Intern Med 2015;175:76-87.

10. Chisholm-Burns MA, Lee JK, Spivey CA, et al. US pharmacists' effect as team members on patient care: systematic review and meta-analyses. Med Care 2010;48:923-933.

11. Ramealho de Oliveira D, Brummel AR, Miller DB. Medication therapy management: 10 years of experience in a large integrated health care system. J Manag Care Pharm 2010;16:185-195.

12. Brummel AR, Soliman AM, Carlson AM, Ramalho de Oliveira D. Optimal diabetes care outcomes following faceto-care medication therapy management services. Popul Health Manag 2013;16:28-34.

13. Brummel A, Carlson A. Comprehensive medication management and medication adherence for chronic conditions. J Manag Care Spec Pharm 2016;22:56-62.

14. Cipolle R, Strand L, Morley P. Pharmaceutical care practice: the clinician's guide, 2nd edition. New York: McGraw Hill, 2004.

15. McInnis T, Webb CE, Strand LM. The patient-centered medical home: integrating comprehensive medication management to optimize patient outcomes, 2nd edition. 2012. www.pcpcc.org/guide/patient-health-through-medicationmanagement\#sthash.Cn2sDgRl.dpuf Accessed June 20, 2015.

Address correspondence to: Holly Budlong, PharmD, PhD Health Outcomes

Fairview Pharmacy Services

711 Kasota Avenue SE Minneapolis, MN 55414

E-mail: hbudlon1@fairview.org 